\title{
Técnica simplificada en la rehabilitación del desdentado
}

\section{Simplified technique for the rehabilitation of the edentulous}

\author{
Biotti Picand J1 , García Nieto JP1
}

\section{RESUMEN}

En esta comunicación se presenta una técnica simplificada de rehabilitación del desdentado total por medio de prótesis removible en que solamente en tres citas clínicas se logra el objetivo del tratamiento. Este logro es posible de ser llevado a cabo por medio del uso de un material fotopolimerizable con el que se realiza la cubeta individual directamente en boca, sin la confección de modelos preliminares, permitiendo de este modo efectuar la toma de impresiones preliminares, definitivas y relaciones intermaxilares en una sola sesión.

Rev. Clin. Periodoncia Implantol. Rehabil. Oral Vol. 7(1); 17-20, 2014.

Palabras clave: Prótesis total, tratamiento del desdentado total, impresiones.

\section{ABSTRACT}

In this paper we present a simplified rehabilitation technique for the edentulous through removable prosthesis that achieve the goal of the treatment in only three clinical appointments. This accomplishment is possible through the use of a photopolymerizable material, performing an impression tray directly in the mouth without making any preliminary models; it thereby enables to take preliminary and definitive impressions, and intermaxillary relations in a single session.

Rev. Clin. Periodoncia Implantol. Rehabil. Oral Vol. 7(1); 17-20, 2014.

Key words: Dentures, edentulous treatment, impression.

\section{INTRODUCCIÓN}

Los odontólogos especialistas que en su práctica diaria efectúan la rehabilitación del desdentado total, requieren de a lo menos seis citas de atención clínica con sus pacientes para realizar las diferentes etapas necesarias para la confección de los aparatos protésicos.

En el común de los casos, las citas en el consultorio implican un tiempo de alrededor de 30 minutos cada una, dependiendo de las habilidades y experiencia del operador algunas de ellas se podrán realizar aproximadamente en 20 minutos.

Las etapas en el procedimiento de la rehabilitación del desdentado total tradicionalmente involucra las siguientes citas clínicas ${ }^{(1)}$.

Primera Cita: Examen, diagnóstico y plan de tratamiento. Impresiones preliminares.

Segunda Cita: Recorte mecánico y funcional de la cubeta individual. Impresiones definitivas.

Tercera Cita: Relaciones cráneo mandibulares, selección de dientes artificiales.

Cuarta Cita: Prueba en boca de los aparatos protésicos en cera.

Quinta Cita: Instalación de los aparatos protésicos.

Sexta Cita: Control pos-instalación.

En esta comunicación, y a través de la presentación de un caso clínico, se propone una técnica simplificada para la rehabilitación del desdentado total, que con una secuencia simple y eficaz logra disminuir el número de las sesiones clínicas para la confección de los aparatos protésicos, circunscribiéndola a solamente tres citas clínicas.

En el procedimiento convencional, en la primera cita el odontólogo toma impresiones preliminares para que en el laboratorio se confeccione un modelos de estudio y cubetas individuales. En la técnica que se desarrolla en esta comunicación, en la primera cita clínica después de efectuado el examen y diagnóstico, se toman las impresiones preliminares con hidrocoloide irreversible (alginato) que es colocado sobre una cubeta de stock y a la vez es cubierto con una lámina de acrílico foto-polimerizable, todo este complejo alginatoacrílico fotopolimerizable se introduce en la boca del paciente y se espera por un lado que el alginato gelifique y además, que el acrílico tome la forma y contornos paraprotésicos, este complejo formado por el alginato gelificado y el acrílico que ha tomado la forma deseada se retira de boca y se lleva por 25 segundos a un horno que por medio de luz visible estroboscópica, permite la polimerización de éste y así se obtiene una cubeta de acrílico rígida que será utilizada para el desarrollo de las siguientes etapas ${ }^{(2)}$.

De esta forma se habrá logrado una disminución de las citas clínicas necesarias para la confección de los aparatos protésicos, de modo tal que el tratamiento quedará circunscrito a solo tres sesiones.

\section{DESARROLLO CLÍNICO DE LA TÉCNICA}

\section{Cita Clínica $\mathbf{N}^{\circ} 1$}

Paciente de sexo masculino que consulta para renovar sus aparatos protésicos por falta de soporte, retención y estabilidad, con múltiples reparaciones y dientes artificiales con severos desgastes.

En el análisis de su cavidad oral se constata una clase III de Angle, con mucosas hiperémicas, lengua saburral, rebordes alveolares residuales disminuidos de tamaño, bajos y redondeados (clase $\mathrm{V}$ de Atwood) $)^{(3)}$.

En esta cita clínica se realizan las impresiones preliminares, confección de cubetas individuales, placas de relación, relaciones intermaxilares y toma de impresiones definitivas.

Las Figuras 1 y 2 nos muestra el instrumental y materiales para la toma de impresiones y el horno que permite efectuar la polimerización del acrílico para confeccionar las cubetas individuales ${ }^{(4)}$.

La Figura 3 nos muestra el alginato colocado en la cubeta de stock y sobre este la lámina de acrílico. Todo este conjunto es introducido en la cavidad oral del paciente, permitiendo de esta forma la toma de la impresión, la cual una vez que ha gelificado el material (alginato),

1. Facultad de Odontología, Universidad de los Andes. Chile.

Correspondencia autor: Jorge Biotti Picand. jbiotti@miuandes.cl. Av. Las Condes 10373 of 46. Las Condes, Santiago, Chile. Trabajo recibido el 20/04/2013. Aprobado para su publicación el 23/06/2013. 
se retira de boca (Figura 4) y se lleva por 25 segundos al horno de fotopolimerización (Figura 5).

Una vez conseguido el endurecimiento del acrílico en el horno de polimerización a través de la luz visible estroboscópica (25 segundos), se procede a su separación y así se obtiene la cubeta acrílica que se utiliza en esta misma cita para la determinación de las relaciones intermaxilares y la toma de las impresiones definitivas (Figuras 6 y 7 ).

En aquellos casos clínicos en que no se haya obtenido una cubeta con el grosor, la fidelidad y extensión adecuadas, el material fotopolimerizable permite adicionar pequeñas porciones sobre la superficie de la impresión ya obtenida (Figura 8), previo a su polimerización en el horno, para así obtener definitivamente un dispositivo-cubeta con los requisitos físicos necesarios.

Una vez retirada la cubeta del horno y separada del alginato, se le da la forma y característica de una placa de relación agregándole un rodete de cera rosa de tal modo que este dispositivo llegue a tener los parámetros estándar ${ }^{(5)}$ para ser usado en la obtención de los registros de las relaciones intermaxilares (Figura 9).

Estas placas de relación confeccionadas de esta forma, se llevan a boca y se evalúa su aspecto estético y funcional(6), para de este modo estar en condiciones de poder obtener los registros de las relaciones intermaxilares (Figura 10).

Una vez logrados los registros intermaxilares, se efectúa la toma de las impresiones definitivas con un material de consistencia mediana, como es el caso de una silicona por condensación, y la particularidad que tendrá en este caso, es que serán impresiones de tipo autogeneradas (autoimpresión), a boca cerrada y manteniendo el registro intermaxilar en el plano vertical( ${ }^{(7)}$ (Figura 11).

En esta misma cita se procede a la selección del color y molde (forma y tamaño) de las piezas dentarias artificiales ${ }^{(8)}$.

El laboratorio dental recibe un complejo impresión-relaciones intermaxilares, con los cuales se confecciona modelos de trabajo y se efectúa su montaje en el articulador, posteriormente se enfilan los dientes artificiales y se confeccionan las prótesis en cera $^{(9)}$.

\section{Cita Clínica $\mathbf{N}^{\circ} 2$ (Figura 12)}

En la cita clínica número dos, se prueban las prótesis en cera con los dientes artificiales, dándole énfasis a la valoración estética y funcional, esto quiere decir que el paciente y el profesional evaluarán el efecto estético de las prótesis en relación a su soporte labial, las distintas líneas medias (facial, dentaria y maxilar), el aspecto estético de la sonrisa, fonación, estabilidad oclusal y correctas relaciones intermaxilares ${ }^{(10)}$.

Una vez obtenida la aprobación final por parte del paciente, como así mismo la satisfacción técnica del profesional, se procede a enviar al laboratorio dental para su proceso de transformación al acrílico.

\section{Cita Clínica $\mathbf{N}^{\circ} 3$ (Figura 13)}

Recibidas por parte del profesional las prótesis en acrílico, en esta cita se proceder a su instalación en boca, la cual debe seguir una secuencia de acciones que en primera instancia consiste en efectuar un análisis riguroso de ambos aparatos protésicos y comprobar, previo a su instalación que estos tengan: Espesor adecuado, bordes redondeados, superficies pulidas y que no existan ángulos agudos ni imperfecciones en las superficies internas ${ }^{(11)}$.

Finalizado este análisis por parte del profesional, es el momento en esta cita clínica efectuar la prueba en boca de las prótesis nuevas y realizar los controles pertinentes en relación a su aspecto estético y funcional, como asimismo, darle énfasis a que comprenda las limitaciones que tienen estos dispositivos en boca ${ }^{(12)}$.

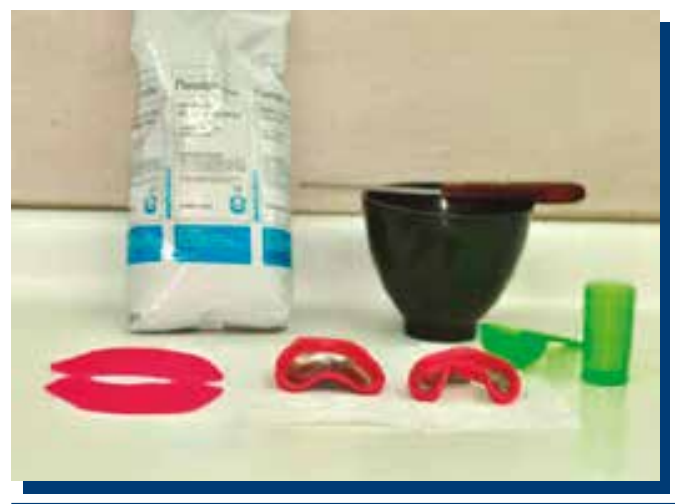

Figura 1.

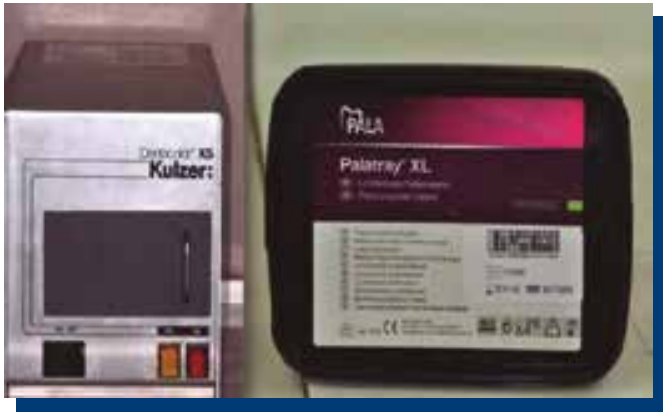

Figura 2.

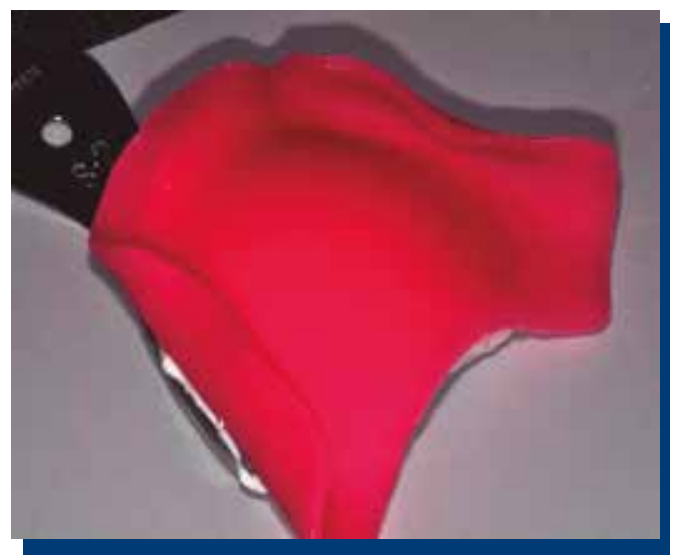

Figura 3.

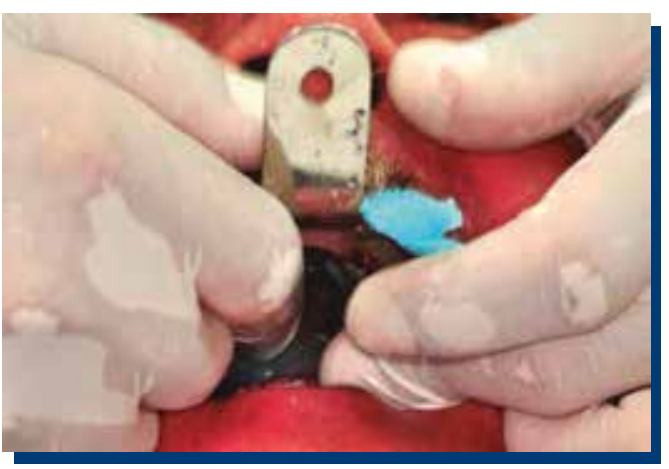

Figura 4. 


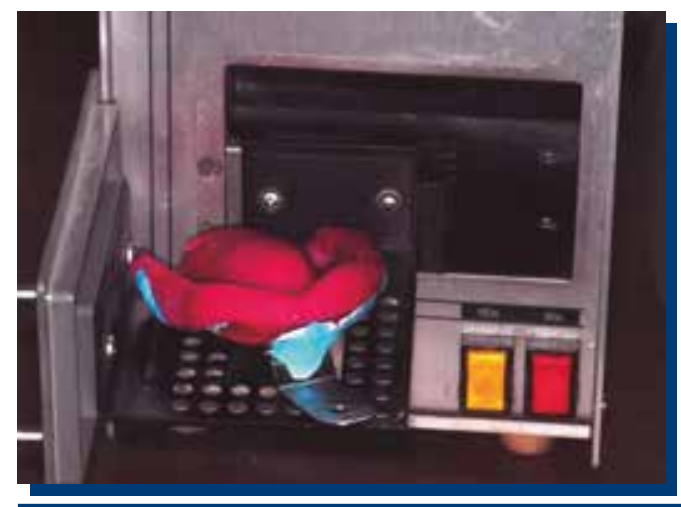

Figura 5.
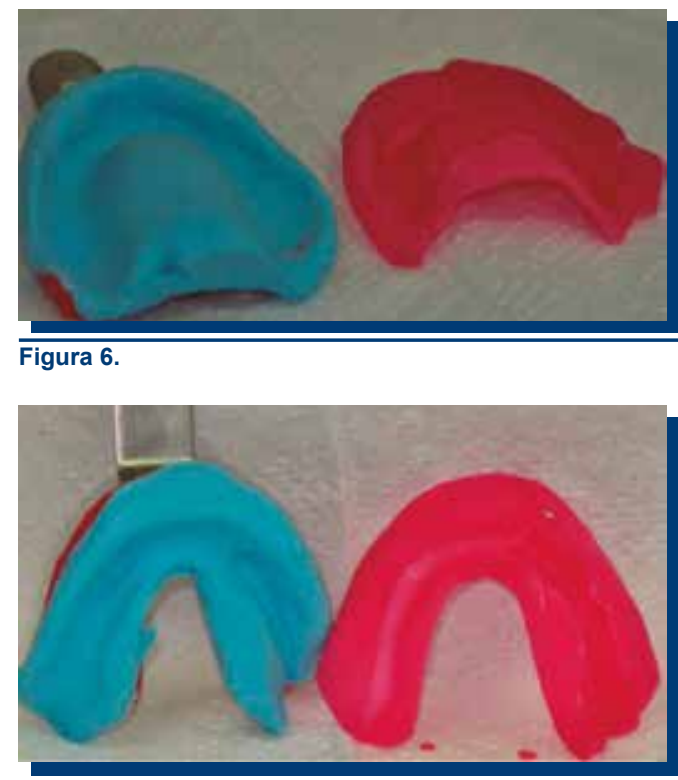

Figura 7.

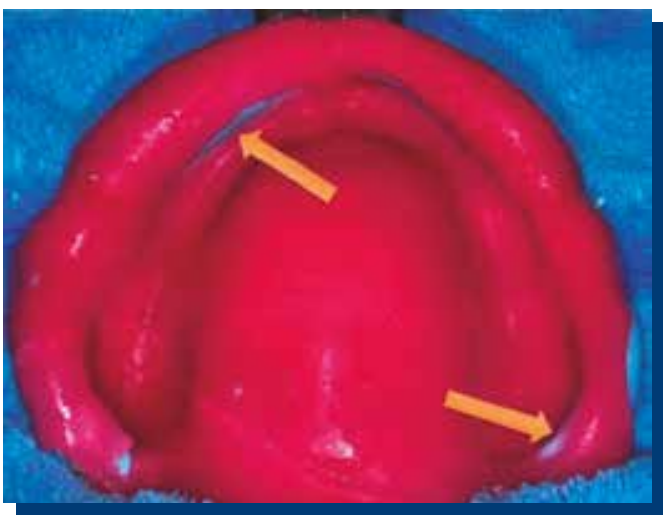

Figura 8.

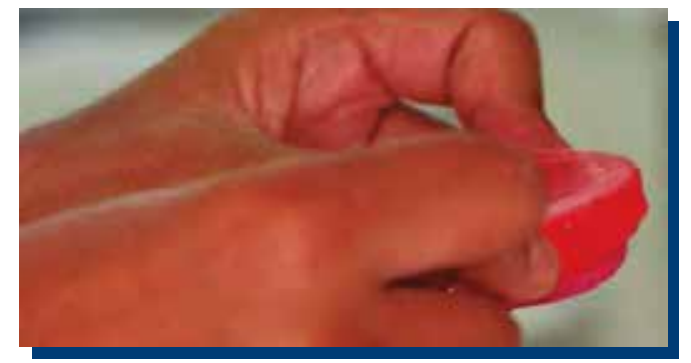

Figura 9.



Figura 10.



Figura 11.

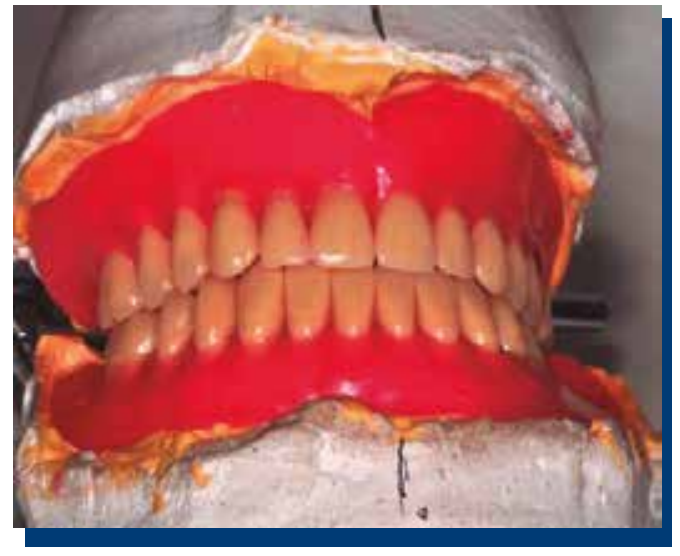

Figura 12.

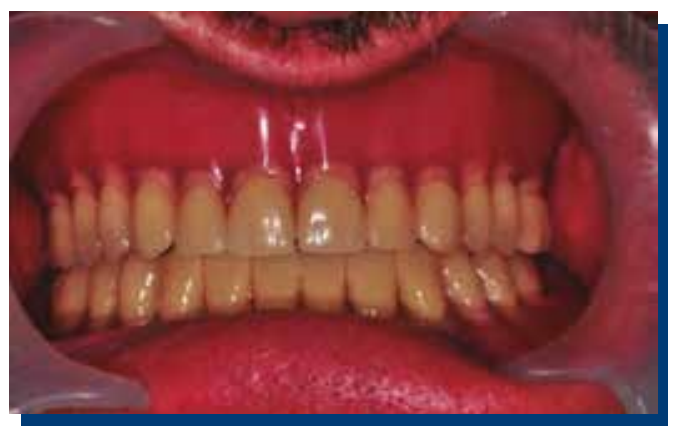

Figura 13. 


\section{COMENTARIO FINAL}

Difundir esta técnica permite al odontólogo especialista tener otra alternativa clínica, que en un menor tiempo (tres sesiones), sin haber descuidado los aspectos técnicos de la rehabilitación del desdentado total se logra una recuperación de éste que cumple con todos los parámetros exigibles, que dicen relación con la estética, retención, soporte, estabilidad y su integración al sistema estomatognático.

\section{CONFLICTO DE INTERESES}

Los autores declaran no presentar conflictos de interés de ningún tipo.

\section{REFERENCIAS BIBLIOGRÁFICAS}

1. Parra N. Prótesis Completas. 1969. Ed. Universitaria.

2. Devlin H, Cash AJ. Mechanical behaviour and structure of light-cured special tray materials. J Dent, 1995; 23(Issue 4): 255-259.

3. Misch CE. Prótesis dental sobre implantes. Capítulo 8. Página 105. Año 2007. Elsevier Mosby.

4. Kulzer catálogo. Modo de empleo. http://www.heraeuskulzer.ru/i/docs/GBA Dentacolor.pdf.

5. Rahn A, Ivanhoe J, Plummer K. Prótesis dental completa. Ed. Panamericana. 2011. Cap. 8. Páginas 132-138.
6. Preti G. Rehabilitación protésica. Tomo 2. Páginas 343-345.

7. Koeck B. Prótesis completas. Ed. Elsevier. 2007. Capítulo 4. Páginas 63-65.

8. García JL. Enfilado dentario bases para la estéticas y la estática en prótesis totales. Ed. Amolca. 2006.

9. Morrow RM, Rudd K, Rhoads J. Procedimientos en el laboratorio dental. Tomo I. Prótesis Completas. Ed. Salvat. 1988.

10. Rufenacht C. Fundamental of esthetics. Ed. Quintessence. 1990. Capítulo 4. Páginas 67-132. 